\title{
O CONHECIMENTO DE POLÍGONOS E POLIEDROS: UMA ANÁLISE DO DESEMPENHO DE ALUNOS DO ENSINO MÉDIO EM EXEMPLOS E NÃO-EXEMPLOS
}

\section{The knowledge of polygons and polyhedrons: an analysis of the students' performance from High School in examples and non-examples}

\author{
Marcelo Carlos de Proença ${ }^{1}$ \\ Nelson Antonio Pirola ${ }^{2}$
}

\begin{abstract}
Resumo: $\mathrm{O}$ artigo pretende mostrar os resultados de uma pesquisa cujo objetivo foi analisar o desempenho de alunos do Ensino Médio na identificação de exemplos e não-exemplos de polígonos e poliedros, tendo em vista os atributos definidores e atributos irrelevantes. Participaram da pesquisa 253 alunos, distribuídos em três séries do Ensino Médio de uma escola pública, que responderam um teste de exemplos e não-exemplos. Posteriormente, foram selecionados seis alunos para serem entrevistados sobre seus conhecimentos a respeito dos exemplos e não-exemplos. Os resultados mostraram que os participantes obtiveram uma média baixa $(M=5,59)$ na tarefa que exigiu a identificação de exemplos e não-exemplos de polígonos e poliedros. A análise das entrevistas mostrou que a maioria dos alunos considerou que o atributo irrelevante dos polígonos analisados não interferiu na resposta. Contudo, evidencia-se, de modo geral, que esses alunos apresentaram um conhecimento conceitual longe do esperado para esse nível de ensino.
\end{abstract}

Palavras-chave: Geometria. Conceitos Geométricos. Polígonos e poliedros. Exemplos e não-exemplos.

\begin{abstract}
The article intends to show the results of research whose objective was to analyse the performance of students from High School in the identification of examples and non-examples of polygons and polyhedrons, intending to define the attributes and irrelevant attributes. In the research 253 students participated distributed into three series from High School in public school who answered a test of examples and non-examples. Later, six students were selected to be interviewed about their knowledge of examples and non-examples. The results showed that the participants had low average $(M=5,59)$ in the homework which demanded the identification of examples and non-examples of polygons and polyhedrons. The analysis of the interviews showed that the most students considered that the irrelevant attribute of polygons analyzed did not interfere in the response. However, it is evident, in general, that these students presented a conceptual knowledge far from the expected for level of education.
\end{abstract}

Keywords: Geometry. Geometry concept. Polygons and polyhedrons. Examples and non-examples.

${ }^{1}$ Licenciado em Matemática. Doutorando em Ensino de Ciências e Matemática. Professor bolsista, Universidade Estadual Paulista "Júlio de Mesquita Filho" - Unesp, campus de Bauru. Bauru, SP, Brasil. <marceloproenca@yahoo.com.br>

${ }^{2}$ Licenciado em Matemática. Doutor em Educação Matemática. Docente, Departamento de Educação, Unesp, campus de Bauru. Bauru, SP, Brasil.<npirola@fc.unesp.br>

${ }^{1}$ Rua Albino Tambara, 5-68, apto. 22, Edifício Buriti

Vila Cidade Universitária - Bauru, SP

17.012-470

199

Ciência \&̊ Educação, v. 17, n. 1, p. 199-217, 2011 
Proença, M. C.; Pirola, N. A.

\section{Introdução}

Nas Propostas Curriculares de Matemática (SÃO PAULO, 1992, 1997) e nos Parâmetros Curriculares Nacionais de Matemática (BRASIL, 1998, 2002), pode-se verificar que a geometria é um assunto presente nos Ensinos Fundamental e Médio. Além disso, a atual Proposta Curricular do Estado de São Paulo (SÃO PAULO, 2008, p. 46) traz a geometria como um dos conteúdos fundamentais, entendendo que "a geometria deve ser tratada ao longo de todos os anos, em abordagem espiralada, o que significa dizer que os grandes temas podem aparecer tanto nas séries do Ensino Fundamental quanto nas do Ensino Médio, sendo que a diferença será a escala de tratamento dada ao tema".

A justificativa por tal presença pode ser verificada por algumas pesquisas (O'DAFFER, 1980; POST, 1980; NOT, 1981 apud PAVANELLO; ANDRADE, 2002) que apontam a geometria como o ramo da matemática mais propício ao desenvolvimento de capacidades intelectuais como a criatividade e a percepção espacial. Para Fainguelernt (1999), a geometria auxilia no desenvolvimento do pensamento espacial e na ativação da intuição, percepção e representação por meio da visualização das figuras. Além disso, o estudo da geometria propicia o desenvolvimento do raciocínio lógico, organização do pensamento, abstração e das capacidades de conjecturar e validar resultados.

Os documentos oficiais citados anteriormente destacam que o ensino de geometria pode ser desenvolvido por meio: do uso de materiais manipulativos (NACARATO, 2005; PAIS, 2002), de jogos (GRANDO, 2007; MENEZES; FOSSA, 2003), da história da matemática (MIGUEL, 1997; SCHEIDE, 2006), da construção, por meio de régua e compasso (PIROLA, 1995; ZUIN, 2003) e manipulação das formas geométricas mais frequentes, da diferenciação entre figuras planas e não planas (PROENÇA; PIROLA, 2006, 2007) e uso de softwares (RIGODANZO; ANGELO, 2004; SILVA, 2003), entre outros - situações que se entende serem importantes na formação dos conceitos presentes nessa área da matemática.

Embora haja muitas possibilidades didáticas voltadas à formação de conceitos geométricos, pesquisas como a de Pais (2002) e Pirola e Brito (2001) salientaram que, no ensino de matemática, tem-se valorizado mais a memorização de fórmulas, regras e definições, o que pouco contribui na formação de conceitos, dificultando o estímulo e a aprendizagem significativa por parte dos alunos. Gonçalez e Brito (2001, p. 226) destacaram que o conhecimento matemático é resultado de uma elaboração mental, sendo que:

Os alunos que recebem o conteúdo matemático em sua forma pronta e acabada tornam-se cada vez mais incapazes de transferir as aprendizagens novas ou de trabalhar com abstrações, dificultando sobremaneira a transferência da aprendizagem de conteúdos escolares para outras situações, diferentes daquela na qual essa aprendizagem ocorreu.

Os Parâmetros Curriculares Nacionais de Matemática (BRASIL, 1998, p. 51) salientam a importância do ensino de conceitos na escola, pois "O aluno desenvolve um tipo especial de pensamento que lhe permite compreender, descrever e representar, de forma organizada, o mundo em que vive". Segundo Klausmeier e Goodwin (1977), ao se desenvolver um conceito, pode-se não apenas resolver atividades simples que exigem a percepção da situação, mas 
generalizar para outros exemplos desse conceito, perceber relações de inclusão que o envolvem com outros, perceber relações de causa e efeito, probabilidade e correlação, e utilizá-lo para resolver problemas que necessitam de um nível de aprendizagem mais formal. Zabala (1998) ressalta que o grau e os níveis de profundidade e compreensão de um conceito são os pontos principais a serem desenvolvidos pelos alunos, permitindo utilizá-lo em diversas situações, não correspondendo, portanto, à mera definição. Para Pais (2002), tal compreensão refere-se ao alcance de níveis satisfatórios de generalidade e abstração. Nesse sentido, percebese que valorizar a memorização arbitrária de fórmulas e regras sem abordar o trabalho em sala de aula sobre os conceitos pode levar o aluno a apresentar dificuldade no desenvolvimento da compreensão para resolver atividades que envolvem matemática.

As avaliações governamentais aplicadas nas instituições de Ensino Básico exigem certas competências e habilidades dos alunos, sendo necessário, entre outras, a compreensão e o conhecimento de conceitos. Por exemplo, o Saresp - Sistema de Avaliação de Rendimento Escolar do Estado de São Paulo - no ano de 2007 (SÃO PAULO, 2007), mostrou que, em geometria, os alunos tiveram um índice indesejável no acerto de questões que exigiam competências e habilidades que deveriam ter sido desenvolvidas na escola. Segundo Pavanello e Andrade (2002, p. 79), a baixa pontuação dos alunos em questões que têm por tema a geometria, conferidas e evidenciadas nos estudos apresentados pelo Governo, "demonstra não serem essas as questões abordadas em sala de aula, ou, na melhor das hipóteses, serem elas trabalhadas de modo bastante precário".

As pesquisas também apontam para o baixo desempenho dos alunos quando o assunto envolve o conhecimento de conceitos em geometria. O estudo de Passos (2000) mostrou que um aluno do Ensino Fundamental, ao ter de resolver o cálculo da área de um triângulo equilátero, o qual estava apoiado na horizontal por um de seus vértices, disse que não era possível, pois a base seria zero, por ser apenas um ponto. No estudo de Proença, Pirola e Quintiliano (2003), alunos do Ensino Médio mostraram pouco conhecimento conceitual sobre figuras planas e não planas ao denominaram a pirâmide de base quadrada de triângulo e o cubo de quadrado.

No trabalho de Proença (2008), alunos do Ensino Médio não superaram a média de cinco pontos, em uma escala de zero a dez, nos testes que exigiram a identificação de polígonos e poliedros e de seus exemplos e não-exemplos. Pirola (1995) também evidenciou desconhecimento, por parte dos alunos do Ensino Fundamental (Ciclo II), de características importantes de figuras geométricas básicas, como triângulos e paralelogramos, apresentando dificuldades na identificação de exemplos e não-exemplos dessas figuras.

Pode-se perceber que é necessário ao aluno desenvolver os conceitos de geometria de forma satisfatória, o que possibilitaria a transferência desses conceitos para a solução de situações-problema.

\section{Aprendizagem e desenvolvimento de conceitos}

Klausmeier e Goodwin (1977, p. 312) elaboraram um Modelo de Aprendizagem e Desenvolvimento de Conceitos (ADC), o qual considera um conceito como uma 
informação ordenada sobre as propriedades de uma ou mais coisas objetos, eventos ou processos - que torna qualquer coisa ou classe de coisas capaz de ser diferenciada de ou relacionada com outras coisas ou classes de coisas.

Para os autores, a palavra conceito é usada para designar tanto os construtos mentais de indivíduos como, também, as entidades públicas identificáveis, que compreendam parte do conteúdo das várias disciplinas. Os conceitos como construtos mentais formam-se de acordo com as experiências de aprendizagem e os padrões maturacionais únicos de cada indivíduo. Conceitos como entidades públicas são definidos como informações organizadas que correspondem aos significados de palavras que estão contidos em dicionários, enciclopédias e outros livros.

Segundo Klausmeier e Goodwin (1977), qualquer conceito pode apresentar, em graus variados, oito características que seriam importantes para o processo de ensino e de aprendizagem na escola básica. Elas seriam:

1 Aprendibilidade: alguns conceitos são aprendidos pelos indivíduos mais facilmente, por apresentarem exemplos perceptíveis (por exemplo, "árvore") do que outros (por exemplo, "átomo" e "eternidade").

2 Utilidade: a utilidade dos conceitos varia no sentido de que alguns podem ser mais usados do que outros para se compreenderem e formarem princípios, sendo que essa variação também ocorre para resolver problemas.

3 Validade: os conceitos tornam-se válidos na medida em que avançam os estudos sobre ele e, também, quando se tornam mais próximos da definição aceita pelos especialistas.

4 Generalidade: grande parte dos conceitos está disposta hierarquicamente e, quanto mais elevado for o lugar do conceito, mais geral ele será em relação aos conceitos subordinados a ele.

5 Importância: um conceito pode facilitar ou ser essencial para formar outros conceitos. Por exemplo, o conceito de perpendicularidade é importante para que o aluno identifique e compreenda o conceito de triângulo retângulo.

6 Estrutura: qualquer conceito, como entidade pública, apresenta uma estrutura caracterizada pela relação com seus atributos definidores. Essa estrutura é denominada "regra conceitual" (afirmativa, conjuntiva, disjuntiva inclusiva, condicional ou bicondicional), que está presente em quase todos os conceitos escolares. Por exemplo, a afirmação "todos os triângulos apresentam três segmentos de retas" apresenta uma relação do conceito de triângulo com seu atributo definidor. Essa é uma regra conceitual do tipo afirmativa.

7 Perceptibilidade de exemplos: a percepção de exemplos de um conceito, às vezes, não é possível por meio dos órgãos dos sentidos. Por exemplo, na Matemática, para o conceito de infinito, não há exemplo observável.

8 Numerosidade de exemplos: os exemplos dos conceitos variam em quantidade: de um único exemplo, como a Lua da Terra, até infinitos, como é o caso dos números naturais.

Por meio dessas oito características e de experiências com alunos da escola básica, foi possível apresentar o Modelo ADC, o qual descreve como se processa o desenvolvimento conceitual, desde a primeira infância até a adolescência, de acordo com quatro níveis cognitivos: concreto, identidade, classificatório e formal. A condição ou capacidade para formar o mesmo conceito em qualquer nível, obrigatoriamente nessa sequência, está nas operações mentais que cada indivíduo é capaz de desenvolver e em termos do que cada um já sabe. 
Nível concreto - Uma pessoa forma o conceito no nível concreto quando reconhece um objeto que foi encontrado em uma ocasião anterior. Segundo Klausmeier e Goodwin (1977, p. 53), as operações mentais necessárias para esse nível são: "prestar atenção a um objeto, discriminá-lo de outros objetos, representá-lo internamente como uma imagem ou traço e manter a representação (lembrar)."

Depois que a pessoa forma o conceito no nível concreto, ela está apta a desenvolver esse conceito em um nível cognitivo mais elevado, o de identidade.

Nível de identidade - Nesse nível, a operação mental (cognitiva) requerida do indivíduo é generalizar que duas ou mais formas do objeto são o mesmo objeto. "A formação no nível de identidade envolve tanto discriminar várias formas de outros objetos, como também generalizar as formas equivalentes" (KLAUSMEIER; GOODWIN, 1977, p. 53). Isso quer dizer que o nível de identidade é inferido quando o indivíduo reconhece um objeto independentemente da sua perspectiva física ou sensorial diferente.

Segundo o modelo, depois que o indivíduo desenvolveu as operações mentais para os níveis anteriores e aprendeu um conceito nesses dois níveis, ele pode desenvolver uma operação mental mais complexa e formar esse conceito ao nível classificatório.

Nível classificatório - A operação cognitiva requerida do indivíduo, nesse nível, é generalizar que dois ou mais exemplos são equivalentes e pertencem à mesma classe de coisas, ou seja, o indivíduo necessita responder a, pelo menos, dois diferentes exemplos de uma classe de objetos como equivalentes.

Indivíduos ainda estão no nível classificatório quando podem classificar corretamente um grande número de instâncias como exemplos e outras como não-exemplos, mas não podem definir a palavra que representa o conceito e também não podem explicar a base da classificação. (KLAUSMEIER; GOODWIN, 1977, p. 54)

Depois de formado o conceito nos três níveis anteriores e tendo formado mais precisamente o conceito no nível classificatório, o indivíduo pode desenvolver as operações mentais para formar o conceito ao nível formal, o mais complexo e de interesse para a atividade escolar.

Nível formal - Um indivíduo forma o conceito nesse nível quando ele consegue fornecer o nome do conceito, definir o conceito em termos de seus atributos definidores, discriminar e nomear seus atributos e diferenciar entre exemplos e não-exemplos, de acordo com os atributos definidores. Em geometria, por exemplo, forma-se o conceito de triângulo equilátero ao nível formal quando o indivíduo o chama de "triângulo equilátero"; identifica seus atributos definidores: três lados iguais; plano, simples, fechado; e três ângulos congruentes, sabendo dizer seus nomes; consegue diferenciá-lo de triângulos isósceles e escalenos (nãoexemplos) porque estes possuem dois lados iguais e os três lados de medidas diferentes, respectivamente.

Para um trabalho em sala de aula, buscando o desenvolvimento de um conceito em cada um desses níveis cognitivos, as oito características citadas anteriormente são importantes. Seria um primeiro passo para obter os atributos definidores, identificar atributos irrelevantes e discriminar exemplos e não-exemplos. Estas três características, segundo Klausmeier e Goodwin (1977), poderiam favorecer a aprendizagem de um determinado conceito. 
Atributos (definidores e irrelevantes) - De acordo com Klausmeier e Goodwin (1977, p. 52), "um atributo é uma característica discriminável de um objeto ou evento que pode assumir valores diferentes, por exemplo, cor, forma, etc." Existem dois tipos de atributos: os atributos definidores e os atributos irrelevantes. Os primeiros correspondem às características ou propriedades que definem um conceito. Por exemplo, o conceito de polígono é definido como uma figura plana, fechada, simples, formada de segmentos de reta. Já os segundos, são as características que podem estar presentes nas figuras, mas que não fazem parte da definição de um conceito. Por exemplo, polígonos com cor interna, hachuras, bordas espessas ou finas, tamanho diferentes, orientação na página etc.

Exemplos e não-exemplos: Os exemplos são as figuras que apresentam todos os atributos definidores exigidos por um determinado conceito. Os não-exemplos são os representantes que não têm todos os atributos definidores desse mesmo conceito. Assim, o conceito de polígono, de acordo com seus atributos definidores, apresenta os triângulos, quadriláteros, pentágonos etc., como exemplos. Já as figuras como o círculo e a circunferência são seus não-exemplos, pois, apesar de serem figuras planas e fechadas, não são formadas de segmentos de reta.

De acordo com Klausmeier e Goodwin (1977), trabalhar com exemplos e não-exemplos no ensino possibilita a redução ou evita os erros ocasionados pela supergeneralização, pela subgeneralização e pela má concepção do indivíduo sobre um conceito. Além disso, conhecer os atributos definidores permite que o aprendiz possa identificar novos exemplos de um conceito e diferenciá-los dos não-exemplos, sendo que o desenvolvimento nos níveis cognitivos possibilita reduzir ou mesmo evitar erros de generalização.

Nesse caso, quando se ensina o conceito de polígono, é de fundamental importância haver um trabalho com as figuras planas e não planas para que os estudantes não façam inferência de forma equivocada de que uma pirâmide é um triângulo e vice-versa. Se, na formação do conceito de triângulo, são apresentados, como exemplos, apenas triângulos eqüiláteros, o aluno pode, também, generalizar incorretamente o conceito por meio de um único tipo de exemplo.

O objetivo do presente estudo foi investigar e analisar o desempenho de alunos do Ensino Médio na identificação de exemplos e não-exemplos de polígonos e poliedros, tendo em vista os atributos definidores e atributos irrelevantes.

\section{Metodologia}

Participaram da pesquisa 253 alunos do Ensino Médio de uma escola pública de uma cidade do interior do Estado de São Paulo, os quais responderam a um teste de exemplos e nãoexemplos elaborado com 24 figuras, contendo exemplos de polígonos, poliedros, figuras que não eram polígonos e dos corpos redondos. O Quadro 1 mostra as figuras que fizeram parte do instrumento de coleta de dados.

O teste foi aplicado nas três séries do Ensino Médio, sendo que o instrumento trazia desenhadas as figuras das três primeiras linhas do Quadro 1. Os participantes tinham de responder a uma alternativa para cada figura: se era polígono, poliedro ou nda (nenhuma das anteriores). Depois de respondido, o pesquisador apresentou as formas geométricas construídas com cartolina (cubo, prisma de base triangular, quadrilátero, círculo, pirâmide de base quadrada e esfera, nessa ordem) para que identificassem a quais das três alternativas corres- 


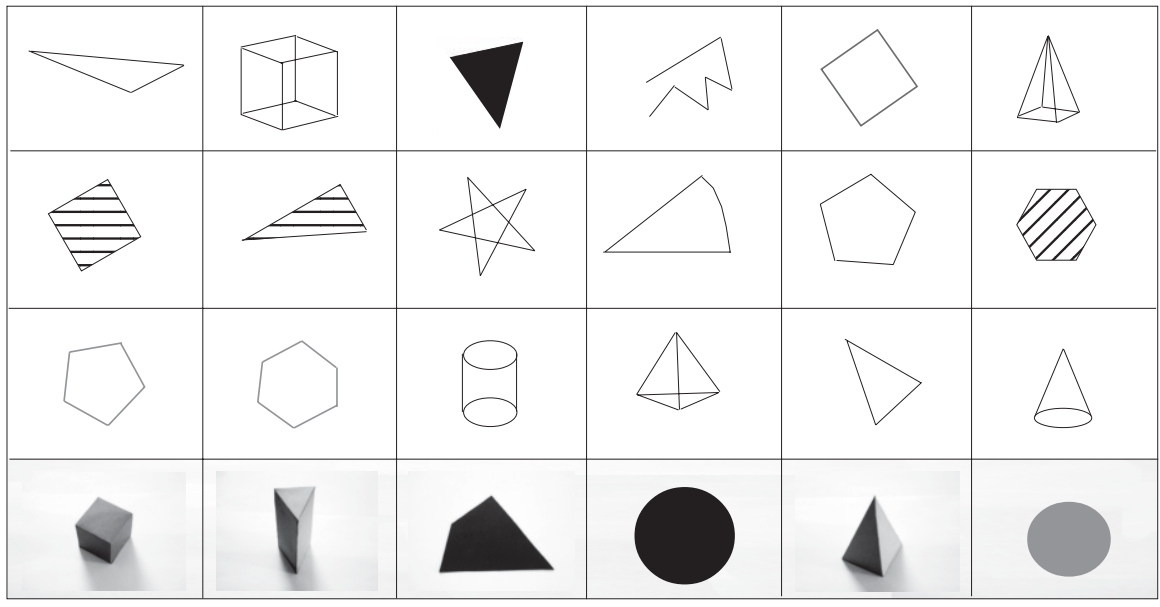

Quadro 1. Figuras geométricas utilizadas na investigação.

Quadro 2. Gênero, idade e média dos alunos que foram entrevistados.

\begin{tabular}{|l|l|c|c|}
\hline Entrevistado/série & Gênero & Idade (anos) & $\begin{array}{c}\text { Média obtida no teste de exemplos e } \\
\text { não-exemplos (escala de 0 a 10 pontos) }\end{array}$ \\
\hline $\mathrm{P} 1\left(1 .{ }^{\circ} \mathrm{C}\right)$ & masculino & 15 & 4,9 \\
\hline $\mathrm{P} 2\left(2 .{ }^{\circ} \mathrm{B}\right)$ & feminino & 17 & 4,9 \\
\hline $\mathrm{P} 3\left(3 .{ }^{\circ} \mathrm{C}\right)$ & masculino & 17 & 4,4 \\
\hline $\mathrm{P} 4\left(1 .{ }^{\circ} \mathrm{A}\right)$ & masculino & 15 & 5,1 \\
\hline $\mathrm{P} 5\left(2 .{ }^{\circ} \mathrm{A}\right)$ & feminino & 16 & 6,6 \\
\hline $\mathrm{P} 6\left(3 .{ }^{\circ} \mathrm{A}\right)$ & feminino & 17 & 6,6 \\
\hline
\end{tabular}

pondiam. No final desse teste, solicitou-se, aos alunos, que apresentassem as dificuldades encontradas para resolvê-lo.

Depois dessa fase, foram escolhidos seis alunos para serem entrevistados, dois de cada série, dos quais três obtiveram média menor que cinco inteiros e três obtiveram média maior que cinco inteiros em uma escala de zero a dez, conforme o Quadro 2.

As entrevistas consistiram no diálogo com os seis alunos, individualmente, sobre cada um dos exemplos e não-exemplos de polígonos e poliedros. O objetivo foi obter dados sobre as dificuldades desses alunos, conseguindo, assim, uma compreensão mais clara dessas dificuldades. De acordo com Bogdan e Biklen (1994), a entrevista pode ser utilizada como um instrumento de coleta de dados descritivos na linguagem do próprio participante, permitindo, assim, que o pesquisador consiga desenvolver de maneira intuitiva uma ideia a respeito do modo como os participantes interpretam aspectos do mundo. 
Proença, M. C.; Pirola, N. A.

Os dados coletados pelo teste de exemplos e não-exemplos foram analisados quantitativamente por meio de teste estatístico para que se verificasse se havia diferença significativa: teste qui-quadrado $\left(c^{2}\right)$ para comparar as porcentagens de acerto por série em cada figura e o teste F (ANOVA) para comparar as médias por série. Além disso, apresentaram-se as dificuldades relatadas pelos alunos. Já os resultados das entrevistas foram analisados qualitativamente, dispondo-os em quadros, verificando, desse modo, o desempenho dos alunos quando identificavam figuras como exemplos e não-exemplos de polígonos e poliedros, bem como se havia interferência do atributo irrelevante. Em seguida, apresentou-se um quadro com as identificações corretas dos seis entrevistados em cada uma das 24 figuras.

\section{Análise do desempenho na identificação dos exemplos e não-exemplos}

A Tabela 1 mostra o desempenho dos participantes, por série e geral, na identificação correta das 24 figuras. Além disso, apresenta-se o teste qui-quadrado $\left(\chi^{2}\right)$, que analisa a significância estatística, sendo que as linhas destacadas em cinza correspondem às figuras que apresentaram diferenças significativas $(\mathrm{p}<0,05)$.

Tabela 1. Desempenho dos participantes em cada figura do teste de exemplos e não-exemplos.

\begin{tabular}{|c|c|c|c|c|c|c|}
\hline \multirow[t]{2}{*}{ Figura } & \multicolumn{4}{|c|}{ Porcentagem } & \multicolumn{2}{|c|}{ Teste qui-quadrado } \\
\hline & $1 .^{\mathrm{a}}$ & $2 .^{a}$ & $3 .^{a}$ & Geral & $\square^{2}{ }_{(2)}$ & p-valor \\
\hline 1 Triângulo & 51,6 & 75,7 & 64,0 & 62,8 & 10,287 & 0,006 \\
\hline 2 Cubo & 38,7 & 52,7 & 55,8 & 48,6 & 5,932 & 0,052 \\
\hline 3 Triângulo (cor interna preta) & 51,6 & 71,6 & 66,3 & 62,5 & 7,850 & 0,020 \\
\hline 4 Figura aberta & 67,7 & 77,0 & 80,2 & 74,7 & 3,988 & 0,136 \\
\hline 5 Quadrado borda espessa & 76,3 & 71,6 & 76,7 & 75,1 & 0,680 & 0,712 \\
\hline 6 Pirâmide base quadrada (desenhada) & 41,9 & 59,5 & 62,8 & 54,2 & 9,015 & 0,011 \\
\hline 7 Quadrado hachurado & 60,2 & 50,0 & 67,4 & 59,7 & 5,046 & 0,080 \\
\hline 8 Triângulo hachurado & 49,5 & 54,1 & 62,8 & 55,3 & 3,281 & 0,194 \\
\hline 9 Figura estrelada & 16,1 & 18,9 & 26,7 & 20,6 & 3,254 & 0,196 \\
\hline 10 Triângulo com um lado circular & 39,8 & 29,7 & 26,7 & 32,4 & 3,812 & 0,149 \\
\hline 11 Pentágono normal & 69,9 & 79,7 & 83,7 & 77,5 & 5,201 & 0,074 \\
\hline 12 Hexágono hachurado & 58,1 & 52,7 & 65,1 & 58,9 & 2,573 & 0,276 \\
\hline 13 Pentágono borda espessa & 72,0 & 71,6 & 82,6 & 75,5 & 3,518 & 0,172 \\
\hline 14 Hexágono borda espessa & 62,4 & 68,9 & 76,7 & 69,2 & 4,335 & 0,114 \\
\hline 15 Cilindro & 35,5 & 14,9 & 22,1 & 24,9 & 9,918 & 0,007 \\
\hline 16 Tetraedro & 48,4 & 71,6 & 66,3 & 61,3 & 10,755 & 0,005 \\
\hline 17 Triângulo isósceles & 58,1 & 59,5 & 57,0 & 58,1 & 0,101 & 0,951 \\
\hline 18 Cone & 16,1 & 12,2 & 17,4 & 15,4 & 0,908 & 0,635 \\
\hline 19 Cubo (cartolina) & 40,9 & 52,7 & 53,5 & 48,6 & 3,551 & 0,169 \\
\hline 20 Prisma base triangular (cartolina) & 60,2 & 77,0 & 65,1 & 66,8 & 5,418 & 0,067 \\
\hline 21 Quadrilátero (cartolina) & 43,0 & 56,8 & 52,3 & 50,2 & 3,351 & 0,187 \\
\hline 22 Círculo (cartolina) & 67,7 & 54,1 & 62,8 & 62,1 & 3,309 & 0,191 \\
\hline 23 Pirâmide base quadrada (cartolina) & 63,4 & 73,0 & 61,6 & 65,6 & 2,576 & 0,276 \\
\hline 24 Esfera (isopor) & 75,3 & 39,2 & 64,0 & 60,9 & 23,042 & 0,000 \\
\hline
\end{tabular}

De acordo com a Tabela 1, as três figuras mais acertadas pelos participantes foram referentes aos polígonos (os dois pentágonos e o quadrado de linha espessa), evidenciando 
uma aprendibilidade desses conceitos. Verificando o índice de acertos dos poliedros, o prisma de base triangular feito com cartolina foi o que apresentou maior porcentagem de acertos $(66,8 \%)$ e os dois cubos (desenhado e de cartolina) tiveram o menor índice de acertos (48,6\%).

Além desse baixo desempenho para esses dois cubos, percebe-se, na Tabela 1, que o teste qui-quadrado $(\chi 2)$ não apontou diferença significativa entre as séries para essas figuras geométricas. Isso indica que a perceptibilidade de exemplos desse poliedro ainda está por se desenvolver nos participantes da pesquisa.

Das figuras em que o teste estatístico apontou diferença significativa, a segunda série foi a que apresentou melhor desempenho nos dois exemplos de polígono (triângulo e triângulo com cor interna) e no exemplo de poliedro (tetraedro). Isso mostra que essa série apresentou melhor aprendibilidade dessas figuras em relação à terceira série, a qual deveria demonstrar maior conhecimento.

Em relação aos não-exemplos, o cone (15,4\%), a figura estrelada (20,6\%) e o cilindro $(24,9 \%)$ foram as menos acertadas pelos participantes. No caso do cilindro, em alguns estudos, mostrou-se que tal figura, muitas vezes, é confundida com o cubo, sendo que os alunos acabam fazendo a representação de um cilindro para indicar que se trata de um cubo (PIROLA; PROENÇA; QUINTILIANO, 2003; MORACO, 2006).

De modo geral, a Tabela 2 mostra a nota média obtida em cada uma das três séries investigadas na pesquisa, a média por gênero e a média dos 253 participantes, a qual foi de $55,9 \%$. Isso indica que esses participantes ainda precisam desenvolver conhecimentos sobre a perceptibilidade de exemplos de polígonos e de poliedros.

O teste estatístico, ANOVA, mostrou que não existiu diferença significativa na nota média por série $(F(2,247)=2,892 ; p=0,057)$ e também mostrou que não houve diferença por gênero na nota média total $(F(1,247)=0,613 ; p=0,434)$. Isso indicou que tais participantes apresentaram um conhecimento conceitual sobre exemplos e não-exemplos de polígonos e de poliedros muito próximo, e que pode ser considerado, segundo as notas médias, dependente de um bom ensino.

A Tabela 3 mostra as dificuldades descritas pelos 253 alunos para realizar o teste aplicado.

Pode-se verificar que a maioria dos participantes relatou que teve dificuldade para identificar os polígonos e os poliedros $(21,7 \%)$, evidenciando a falta de domínio da estrutura desses conceitos. Uma porcentagem muito pequena de participantes relatou que teve dificuldades com os atributos irrelevantes $(3,6 \%)$, situação essa relacionada às respostas que evidenciaram dúvidas sobre a figura "ser pintada" (interior preto) e outras serem "figuras listradas" (hachura).

Tabela 2. Desempenho geral (nota média) dos participantes por série e gênero.

\begin{tabular}{|c|c|c|c|c|c|c|c|c|c|}
\hline \multirow{2}{*}{ Série } & \multicolumn{3}{|c|}{ Masculino } & \multicolumn{3}{|c|}{ Feminino } & \multicolumn{3}{|c|}{ Total } \\
\hline & $\mathrm{N}^{\circ}$ & Média & DP & $\mathrm{N}^{\circ}$ & Média & DP & $\mathbf{N}^{\circ}$ & Média & DP \\
\hline $1^{a}$ & 35 & 5,26 & 1,72 & 58 & 5,27 & 1,56 & 93 & 5,27 & 1,61 \\
\hline $2^{a}$ & 26 & 5,43 & 2,03 & 48 & 5,69 & 2,12 & 74 & 5,60 & 2,07 \\
\hline $3^{a}$ & 36 & 6,42 & 2,12 & 50 & 5,55 & 2,22 & 86 & 5,92 & 2,21 \\
\hline Total & 97 & 5,74 & 2,009 & 156 & 5,49 & 1,96 & 253 & 5,59 & 1,98 \\
\hline
\end{tabular}


Proença, M. C.; Pirola, N. A.

Tabela 3. Porcentagem de participantes por série de acordo com as dificuldades que relataram sobre a realização do teste de exemplos e não-exemplos.

\begin{tabular}{lrrrr}
\hline \multicolumn{1}{c}{ Dificuldades relatadas no teste } & 1. $^{\text {a }}$ série & 2. $^{\text {a }}$ série & 3. $^{\text {a }}$ série & Total \\
\hline Polígonos/exemplos & 1,1 & 0,0 & 1,2 & 0,8 \\
Poliedros/exemplos & 5,4 & 4,1 & 7,0 & 5,5 \\
Identificar polígono/poliedro & 26,9 & 12,2 & 24,4 & 21,7 \\
Atributos irrelevantes & 4,3 & 4,1 & 2,3 & 3,6 \\
Não sabe/lembra geometria & 17,2 & 14,9 & 12,8 & 15,0 \\
Não respondeu & 32,3 & 50,0 & 44,2 & 41,5 \\
Nenhuma & 12,9 & 14,9 & 8,1 & 11,9 \\
Total & 100,0 & 100,0 & 100,0 & 100,0 \\
\hline
\end{tabular}

\section{Análise das entrevistas}

Buscou-se, primeiramente, analisar as respostas dos alunos a respeito de um exemplo de polígono (triângulo com cor interna preta) e de um exemplo de poliedro (cubo desenhado). Os quadros que serão apresentados mostram duas colunas com os resultados sobre cada figura. Na coluna resposta assinalada no teste, destaca-se o que o entrevistado tinha assinalado no teste: polígono, poliedro ou nda. Essa resposta foi comparada a suas explicações na coluna resposta no diálogo da entrevista, na qual foram destacados diálogos ocorridos na entrevista que possibilitavam perceber o que os alunos conheciam.

O Quadro 3 mostra os diálogos das entrevistas sobre a identificação do exemplo de polígono, triângulo, cujo atributo irrelevante é a cor preta. No geral, todos responderam na entrevista que se tratava de um polígono, dizendo também o tipo da figura, um triângulo, o que evidencia um conhecimento da estrutura dessa figura. O atributo irrelevante - cor preta gerou dificuldades na identificação desse exemplo apenas para o participante P5. Para Klausmeier e Goodwin (1977), na formação conceitual, o atributo irrelevante como, por exemplo, a cor da figura, não faz parte das informações ordenadas sobre as propriedades de um conceito, ou seja, de sua estrutura.

$\mathrm{Na}$ definição de polígono em geometria, conforme Barbosa (1985) e Dolce e Pompeo (1993), levou-se em consideração a linha poligonal, ou seja, os segmentos de reta, e não sua região interna. Em contrapartida, alguns autores de livros didáticos têm apresentado definições que caracterizam polígonos sendo a linha poligonal mais a região interna. Possivelmente, isso seja uma postura assumida para uma abordagem posterior do assunto de áreas.

$\mathrm{Na}$ visão de Barbosa (1985), é necessário fazer uma convenção, dizendo aos alunos que "nós iremos tomar a liberdade de usar expressões do tipo a área de um quadrado quando queremos dizer realmente 'a área da região poligonal cuja fronteira é um quadrado"' (BARBOSA, 1985, p. 176).

O Quadro 4 mostra o diálogo das entrevistas na identificação do exemplo de poliedro, cubo. Observa-se que P4, P5 e P6 foram os únicos que identificaram o cubo como um tipo de poliedro nas duas fases da pesquisa. O participante P2 havia repetido a resposta dada na primeira fase, mudando-a depois de questionada pelo pesquisador, pois tinha dito que cubo era quadrado. 
Quadro 3. Respostas dos participantes sobre o triângulo com cor interna preta.

\begin{tabular}{|c|c|}
\hline \multicolumn{2}{|c|}{$\begin{array}{l}\text { Figura: triângulo } \\
\text { Atributo irrelevante: cor interna preta }\end{array}$} \\
\hline $\begin{array}{c}\text { Resposta } \\
\text { assinalada no teste }\end{array}$ & Resposta no diálogo da entrevista \\
\hline Poliedro & $\begin{array}{l}\text { P: "Essa figura é polígono, poliedro ou nda?" } \\
\text { P1: "Polígono." } \\
\text { P: "Como você chegou à conclusão que era polígono?" } \\
\text { P1: "Triângulo". } \\
\text { P: "E essa cor preta?" } \\
\text { P1: "Acho que não tem nada haver". }\end{array}$ \\
\hline Polígono & $\begin{array}{l}\text { P: "Essa figura é polígono, poliedro ou nda?" } \\
\text { P2: "Polígono". } \\
\text { P: "E essa cor preta?" } \\
\text { P2: "A cor não influencia em nada." } \\
\text { P: "Que figura é essa?" } \\
\text { P2: "Triângulo." }\end{array}$ \\
\hline Polígono & $\begin{array}{l}\text { P: "Essa figura é polígono, poliedro ou nda?" } \\
\text { P3: "Polígono. Triângulo." } \\
\text { P: "E essa cor preta você levou em consideração?" } \\
\text { P3: "A cor preta não." } \\
\text { P: "Você acha que essa cor preta interferiu na sua resposta?" } \\
\text { P3: "(Não, balançando a cabeça)." }\end{array}$ \\
\hline Polígono & $\begin{array}{l}\text { P: "Essa figura é polígono, poliedro ou nda?" } \\
\text { P4: "Polígono." } \\
\text { P: "Por quê?" } \\
\text { P4: "Porque é plano igual à primeira (referindo-se ao triângulo escaleno)." } \\
\text { P: "E a cor preta?" } \\
\text { P4: "Ah, não faz diferença eu acho. O que vale é o formato." }\end{array}$ \\
\hline Polígono & $\begin{array}{l}\text { P: "Essa figura é polígono, poliedro ou nda?" } \\
\text { P5: "Acho que é polígono. Triângulo." } \\
\text { P5: "Não. Acho que é nda." } \\
\text { P: “O que está te levando a pensar que não é nem polígono nem poliedro?" } \\
\text { P5: "É porque..." } \\
\text { P: "Essa cor preta te atrapalhou em alguma coisa para pensar?" } \\
\text { P5: "Sim." } \\
\text { P: “O que é um polígono?" } \\
\text { P5: "São figuras planas. São...ai, esqueci...São formados por segmentos de reta, } \\
\text { aqui no caso, triângulo." } \\
\text { P: "Que figura é essa?" } \\
\text { P5: "Triângulo." } \\
\text { P: "Então essa figura é o quê?" } \\
\text { P5: "Um polígono." }\end{array}$ \\
\hline Polígono & $\begin{array}{l}\text { P: "Essa figura é polígono, poliedro ou nda?" } \\
\text { P6: "Polígono." } \\
\text { P: "Você levou em consideração essa cor preta para assinalar polígono?" } \\
\text { P6: "Não." } \\
\text { P: "O que te levou a assinalar polígono?" } \\
\text { P6: "Pra mim ele é plano... porque ele não tem perspectiva em 3D... não dá pra ver } \\
\text { se ele tem profundidade, volume." } \\
\text { P: "Essa figura tem nome?" } \\
\text { P6: "Triângulo. Três lados." }\end{array}$ \\
\hline
\end{tabular}


Proença, M. C.; Pirola, N. A.

Quadro 4. Respostas dos participantes sobre o cubo.

\begin{tabular}{|c|c|}
\hline Figura:cubo & \\
\hline $\begin{array}{c}\text { Resposta } \\
\text { assinalada no teste }\end{array}$ & Resposta no diálogo da entrevista \\
\hline Polígono & $\begin{array}{l}\text { P: "Essa figura é polígono, poliedro ou nda?" } \\
\text { P1: "Poliedro." } \\
\text { P1: "Cubo." }\end{array}$ \\
\hline $\mathrm{Nda}$ & $\begin{array}{l}\text { P: "Essa figura é polígono, poliedro ou nda?" } \\
\text { P2: "Poliedro." } \\
\text { P2: "É um cubo." } \\
\text { P: "Se é um cubo, continua sendo nda?" } \\
\text { P2: "Não, é poliedro." } \\
\text { P: "Por quê?" } \\
\text { P2: "Porque tem mais de cinco lados." }\end{array}$ \\
\hline Polígono & $\begin{array}{l}\text { P: "Essa figura é polígono, poliedro ou nda?" } \\
\text { P3: "Poliedro. Uma figura tridimensional." }\end{array}$ \\
\hline Poliedro & $\begin{array}{l}\text { P: "Essa figura é polígono, poliedro ou nda?" } \\
\text { P4: "É poliedro." } \\
\text { P4: "Porque ela tem dimensão." }\end{array}$ \\
\hline Poliedro & $\begin{array}{l}\text { P: "Essa figura é polígono, poliedro ou nda?" } \\
\text { P5: "Poliedro." }\end{array}$ \\
\hline Poliedro & $\begin{array}{l}\text { P: "Essa figura é polígono, poliedro ou nda?" } \\
\text { P6: "Poliedro." }\end{array}$ \\
\hline
\end{tabular}

De modo geral, o Quadro 5 mostra a identificação, nas entrevistas, das figuras que eram exemplos e não-exemplos de polígono e de poliedro pelos participantes. A letra "X" indica que houve uma identificação correta. Já o sinal ".” mostra que o participante não identificou corretamente a figura. Observa-se que a "figura estrelada" e a "esfera" foram identificadas como não-exemplos de polígono e poliedro apenas por P3, o qual, juntamente com P1, foram os entrevistados que menos erraram na identificação de exemplos e nãoexemplos.

O Quadro 5 mostra também que, de todas as figuras que apresentavam atributos irrelevantes, o "quadrado de borda espessa" não foi identificado por P2. De maneira geral, a análise das entrevistas mostrou que os atributos irrelevantes presentes em algumas figuras do teste não atrapalharam na identificação correta pelos alunos.

P: "Essa figura [hexágono hachurado] é polígono, poliedro ou nda?"

P5: "Acho que é polígono."

P5: "Ai esses risquinhos pra atrapalhar! Acho que é polígono."

P: "Que figura é essa?"

P5: "Um hexágono." 
O participante P2 não errou na identificação do "quadrado de borda espessa" por causa do atributo irrelevante mas pelo desconhecimento da sua estrutura, sendo a de uma classe mais geral, a de polígonos.

\section{P: "Por que você acha que é nda?" \\ P2: "Porque é um quadrado. Um quadrado não é polígono e nem poliedro?"}

Pode se ter uma ideia dessa dificuldade no Quadro 5, pois P2 apresentou um índice de 13 identificações incorretas. Um fato constatado durante as entrevistas foi o de que, para P2, o critério para identificar polígonos e poliedros era que, se a figura tivesse até cinco lados, seria um polígono, mais de cinco, um poliedro. Nesse caso, para o cubo, considerou como poliedro, tendo em vista essa ideia. No entanto, foi o único participante que conseguiu identificar o "círculo" como um não-exemplo de polígono e poliedro, pois identificou um atributo definidor, respondeu: "não tem lado".

Quadro 5. Respostas dos participantes na entrevista.

\begin{tabular}{|c|c|c|c|c|c|c|}
\hline \multirow{2}{*}{ Figura } & \multicolumn{6}{|c|}{$\begin{array}{l}\text { Identificação da figura como sendo exemplo ou não-exemplo } \\
\text { de polígono ou de poliedro }\end{array}$} \\
\hline & P1 & $\mathbf{P 2}$ & P3 & P4 & P5 & P6 \\
\hline Triângulo & $x$ & $x$ & $x$ & $x$ & $x$ & $x$ \\
\hline Cubo & $x$ & $\mathrm{x}$ & $x$ & $\mathrm{x}$ & $x$ & $x$ \\
\hline Triângulo (cor interna preta) & $x$ & $x$ & $x$ & $x$ & $x$ & $x$ \\
\hline Figura aberta & $\mathrm{X}$ & $x$ & $x$ & $\mathrm{x}$ & $x$ & $\mathrm{X}$ \\
\hline Quadrado borda espessa & $\mathrm{x}$ & - & $x$ & $x$ & $x$ & $\mathrm{X}$ \\
\hline Pirâmide base quadrada (desenhada) & $x$ & - & $x$ & $x$ & $x$ & $x$ \\
\hline Quadrado hachurado & $x$ & $x$ & $x$ & $x$ & $x$ & $x$ \\
\hline Triângulo hachurado & $x$ & $x$ & $x$ & $x$ & $x$ & $x$ \\
\hline Figura estrelada & - & - & $x$ & - & - & - \\
\hline Triângulo com um lado circular & $x$ & - & $x$ & $x$ & - & - \\
\hline Pentágono normal & $x$ & - & $x$ & $\mathrm{x}$ & $x$ & $\mathrm{X}$ \\
\hline Hexágono hachurado & $x$ & $x$ & $x$ & $\mathrm{x}$ & $x$ & $x$ \\
\hline Pentágono borda espessa & $\mathrm{x}$ & - & $x$ & $\mathrm{x}$ & $\mathrm{X}$ & $x$ \\
\hline Hexágono borda espessa & $x$ & $\mathrm{x}$ & $x$ & $\mathrm{x}$ & $x$ & $x$ \\
\hline Cilindro & $\mathrm{X}$ & - & - & $\mathrm{x}$ & - & - \\
\hline Tetraedro & $\mathrm{x}$ & - & $\mathrm{x}$ & $\mathrm{x}$ & $\mathrm{x}$ & $\mathrm{x}$ \\
\hline Triângulo isósceles & $\mathrm{x}$ & $\mathrm{x}$ & $x$ & $\mathrm{x}$ & $x$ & $x$ \\
\hline Cone & $x$ & $\mathrm{x}$ & - & - & - & $\mathrm{X}$ \\
\hline Cubo (cartolina) & $x$ & - & $x$ & $\mathrm{x}$ & $\mathrm{X}$ & $x$ \\
\hline Prisma base triangular (cartolina) & $x$ & - & $x$ & $\mathrm{x}$ & $\mathrm{x}$ & $\mathrm{x}$ \\
\hline Quadrilátero (cartolina) & $x$ & - & $x$ & $\mathrm{x}$ & $x$ & $x$ \\
\hline Círculo (cartolina) & - & $\mathrm{x}$ & - & - & - & - \\
\hline Pirâmide base quadrada (cartolina) & $\mathrm{x}$ & - & $\mathrm{x}$ & $\mathrm{x}$ & $x$ & $x$ \\
\hline Esfera (isopor) & - & - & $x$ & - & - & - \\
\hline
\end{tabular}




\section{Conclusão e considerações finais}

O objetivo da presente pesquisa foi investigar e analisar o desempenho de alunos do Ensino Médio na identificação de exemplos e não-exemplos de polígonos e poliedros, considerando os atributos definidores e os atributos irrelevantes.

A média de acertos dos 253 participantes da pesquisa, no teste de exemplos e nãoexemplos, foi de 5,59 (DP = 1,98), em uma escala de zero a dez. Não foram encontradas diferenças significativas $(p=0,057)$ entre as médias das notas de cada série. Esse resultado mostra o pouco conhecimento que tinham na identificação de exemplos e não-exemplos de polígonos e poliedros e, consequentemente, para diferenciar figuras que não eram polígonos e figuras pertencentes à classe dos corpos redondos.

Além disso, em relação às figuras que apresentaram diferenças significativas, notou-se uma discrepância entre os acertos das três séries, sendo que a segunda série apresentou melhor desempenho nos dois exemplos de polígono - triângulo e triângulo com cor interna - e no exemplo de poliedro - tetraedro.

A análise das entrevistas permitiu verificar que os seis entrevistados não tiveram dificuldades para identificar, em dois casos específicos, o triângulo como exemplo de polígono e o cubo como exemplo de poliedro. Além disso, ficou evidente que os atributos irrelevantes quase não geraram obstáculo aos alunos.

Desse modo, de acordo com os resultados do teste e das entrevistas, podem-se destacar algumas dificuldades que os participantes tiveram na identificação de exemplos e nãoexemplos de polígono e poliedro: (a) diferenciação entre uma figura plana (bidimensional) e uma figura não plana (tridimensional); (b) conhecimento dos atributos definidores de polígonos e de poliedros; (c) perceber a relação entre um polígono e um poliedro, no que diz ao primeiro compor as faces do segundo; e (d) diferenciar polígonos de figuras que apresentam alguma linha curva como, por exemplo, círculo.

Isso indica que polígonos e poliedros devem ser devidamente trabalhados em sala de aula. Além disso, figuras como "figura estrelada", cilindro, círculo e esfera, não-exemplos desses dois conceitos, precisam ser melhor exploradas na educação básica, pois foram aquelas que os alunos apresentaram menor frequência na identificação.

Destaca-se que o teste aplicado utilizou figuras desenhadas e figuras construídas com material (cartolina). É importante ressaltar que há a situação de que figuras planas construídas com algum tipo de material podem induzir os alunos a pensarem que se trata de figuras espaciais. Nesta pesquisa, os dados coletados mostraram que, por exemplo, o círculo, construído com cartolina, foi identificado por cinco alunos entrevistados como sendo polígono e, por um aluno, como nda (nenhuma das anteriores). Nesse caso, ninguém o confundiu com, por exemplo, cilindro, não denominando, assim, de poliedro.

Cabe, assim, em sala de aula, propiciar aos estudantes o entendimento de que, por exemplo, um triângulo de papel é uma representação desse conceito, e que é necessário para fins de aprendizagem. Silveira (2006) mostrou que o conceito matemático está sempre em estado de devir, na perspectiva do aluno, mesmo que esse conceito seja considerado imutável do ponto de vista do rigor matemático. Para essa autora, "o conceito, antes de ser interpretado pelo aluno, obedece às exigências e à lógica da Matemática; após a interpretação, depende da própria lógica do aluno" (SILVEIRA, 2006, p. 47). 
Refletindo sobre o Modelo de Aprendizagem e Desenvolvimento de Conceitos (ADC), utilizado como referencial teórico neste estudo, e sobre os resultados apresentados, podemos tecer algumas considerações sobre os termos que compõem essa teoria e seus significados e o trabalho em sala de aula que pode ser realizado para melhorar a aprendizagem de polígonos e de poliedros. Destaca-se que várias pesquisas utilizaram esse referencial teórico, articulando-o, muitas vezes, com outras teorias, o que possibilitou compor um quadro teórico mais específico para analisar e discutir dados que, de alguma forma, envolviam o conhecimento conceitual. Trata-se de trabalhos que utilizam a teoria ADC para analisar diferentes aspectos da aprendizagem de conteúdos da matemática escolar (PIROLA, 1995; BRITO, 1996a, 1996b; BRITO; PIROLA; LIMA, 1997; CORREA et al., 1998; REZI, 2001; QUINTILIANO, 2005; SORMANI JUNIOR, 2006; MORACO, 2006; PROENÇA, 2008).

É importante destacar que os termos em estudo, como atributos definidores, atributos irrelevantes, exemplos e não-exemplos, podem fazer parte do vocabulário de sala de aula dos alunos na aprendizagem de polígono e de poliedro. Sua utilização pode ajudá-los a delimitarem melhor as características de cada um, de um em relação ao outro e de cada um em relação a outras figuras geométricas, como não-polígonos e corpos redondos, para diferenciação.

Quando um aluno é levado a analisar, por exemplo, as características comuns de um conjunto de figuras planas, buscando a identificação de polígonos, o fato de procurar por atributos definidores pode ajudá-lo a desenvolver um pensamento que objetiva encontrar particularidades. Desse modo, é possível partir de exemplos e não-exemplos para formalizar os exemplos de polígonos constituintes de tal conjunto. Sabendo-se o que é, pode-se diferenciar o que não é.

Além disso, se há uma compreensão, por parte do aluno, de que o conceito de polígono depende de seus atributos definidores, e não de atributos irrelevantes, a posição em que, por exemplo, um quadrado se encontra desenhado no papel acaba por ser entendida como irrelevante para caracterizar essa figura como quadrado. A posição em que se encontra o quadrado de borda espessa (Quadro 1), por exemplo, leva muitos alunos a defini-lo como losango, sendo que o correto é o de se atentar para as características, isto é, aos atributos definidores.

No caso do ensino em sala de aula, esse modelo (ADC) apresenta oito características que um determinado conceito pode ter: aprendibilidade, validade, utilidade, generalidade, importância, estrutura, perceptibilidade de exemplos e numerosidade de exemplos. Esse conhecimento pode ser utilizado pelo professor para delimitar o conceito de polígono e de poliedro e poder exercer um ensino que favoreça a aprendizagem significativa dos alunos.

Assim, algumas indicações para a elaboração de um trabalho em sala de aula, que se podem sugerir, seriam referentes à realização inicial de uma delimitação desses conceitos em termos de sua estrutura, ou seja, de seus atributos definidores. Posteriormente, pode-se elencar um conjunto de exemplos de cada um e um conjunto de não-exemplos para organizar atividades de identificação e discriminação de figuras que são polígonos e não-polígonos pelos alunos, assim como de poliedros e outras figuras espaciais.

Uma atividade que poderia ser desenvolvida para se alcançar esse objetivo seria o professor desenhar quadrados e retângulos e solicitar aos alunos que destaquem as características (atributos definidores) de cada uma. Com isso, o aluno poderá perceber que existem algumas características comuns entre quadrado e retângulo e outras que os diferenciam. Por exemplo: embora os quadrados e retângulos possuam ângulos retos, os retângulos, não necessariamente, devem possuir lados congruentes. Essas mesmas características podem ser relacio- 
Proença, M. C.; Pirola, N. A.

nadas com as do paralelogramo para que os alunos concluam que o quadrado e o retângulo são exemplos de paralelogramos.

Além disso, pode-se trabalhar de maneira específica, ou seja, estabelecer um conjunto de triângulos (equilátero, isósceles ou escaleno) com diferentes tamanhos para verificar se o aluno percebe que se trata da mesma figura. Situação semelhante pode ser feita para quadrados, pentágonos etc. Pode-se, ainda, estruturar essa atividade com as figuras sendo formadas por outros atributos irrelevantes, para verificar se os alunos os levam em consideração para defini-los.

Desse modo, ao mesmo tempo em que os alunos vão se apropriando desses elementos, podem se propor atividades em que os estudantes relacionem os atributos definidores com exemplos de polígonos e exemplos de poliedros. Assim, tarefas que levem os alunos a relacionarem a descrição de atributos definidores com suas respectivas figuras podem ser um importante exercício de aprendizagem. O trabalho de planificação e construção de poliedros também auxilia a diferenciar polígonos de poliedros por meio da análise dos atributos definidores.

Contudo, segundo os resultados apresentados nesta pesquisa e de acordo com as indicações/sugestão de trabalho com a formação conceitual em geometria, acredita-se que servirá como um ponto importante para se repensar o trabalho em sala de aula e, consequentemente, a formação de professores de matemática.

\section{Referências}

BARBOSA, J. L. M. Geometria euclidiana plana. Rio de Janeiro: Sociedade Brasileira de Matemática, 1985. (Coleção Fundamentos da Matemática Elementar).

BOGDAN, R.; BIKLEN, S. Investigação qualitativa em educação: uma introdução à teoria e aos métodos. Porto: Porto Editora, 1994. (Ciências da Educação).

BRASIL. Secretaria de Educação Média e Tecnológica. Parâmetros Curriculares Nacionais: Ensino Médio. Brasília: MEC/SEMTEC, 2002.

. Secretaria de Ensino Fundamental. Parâmetros Curriculares Nacionais. Brasília: SEF/MEC, 1998.

BRITO, M. R. F. Um estudo sobre a aquisição de conceito de triângulo em crianças de $4^{a} \mathrm{e}$ $5^{\mathrm{a}}$ séries do $1^{\mathrm{o}}$ grau. In: SIMPÓSIO DE PESQUISAS BRASILEIRAS EM DESENVOLVIMENTO SÓCIO-COGNITIVO, 1., 1996, Rio de Janeiro. Anais... Rio de Janeiro: UERJ, 1996a. 1 cd-rom.

. O ensino e a formação de conceitos em sala de aula. In: NOVAES, M. H.; BRITO, M. R. F. (Org.). Psicologia na educação: articulação entre pesquisa, formação e prática pedagógica. São Paulo: ANPEPP, 1996b. p. 73-93.(Coletâneas da ANPEPP, 5).

.; PIROLA, N. A.; LIMA, V. S. Concept formation and representation: a study about triangles in first grade students. In: CONFERENCE OF THE INTERNATIONAL GROUP FOR THE PSYCHOLOGY OF MATHEMATICS EDUCATION, 21., 1997, Lahti, Finland. Proceedings... Lahti: IGPME, 1997. p. 221. 
O conhecimento de polígonos e poliedros: ...

CORREA, J.; SPINILLO, A. G.; BRITO, M. R. F.; MORO, M. L. F. O desenvolvimento de conceitos matemáticos: temas de interesse para a educação matemática. In: MOURA, M. L. S. de; CORREA, J.; SPINILLO, A. G. (Orgs.). Pesquisas brasileiras em psicologia do desenvolvimento. Rio de Janeiro: EDUERJ, 1998. p. 73-110.

DOLCE, O.; POMPEO, J. N. Fundamentos de matemática elementar, 9: geometria plana. 5. ed. São Paulo: Atual, 1993.

FAINGUELERNT, E. K. Educação matemática: representação e construção em geometria. Porto Alegre: Artes Médicas, 1999.

GONÇALEZ, M. H. C. C.; BRITO, M. R. F. A aprendizagem de atitudes positivas em relação à Matemática. In: BRITO, M. R. F (Org.). Psicologia da Educação Matemática: teoria e pesquisa. Florianópolis: Insular, 2001. p. 221-234.

GRANDO, R. C. Concepções quanto ao uso de jogos no ensino da matemática. Revista de Educação Matemática, São Paulo, v. 10, n. 12, p. 43-50, 2007.

KLAUSMEIER, H. J.; GOODWIN, W. Manual de psicologia educacional: aprendizagem e capacidades humanas. São Paulo: Harper \& Row, 1977.

MENEZES, J. E.; FOSSA, J. A. As razões para usar o jogo no contexto ensinoaprendizagem da matemática. In: SEMINÁRIO INTERNACIONAL DE PESQUISA EM EDUCAÇÃO MATEMÁTICA, 2., 2003, Santos. Anais... Santos: SBEM, 2003. 1 cd-rom.

MIGUEL, A. As potencialidades pedagógicas da história da matemática em questão: argumentos reforçadores e questionadores. Zetetiké, Campinas, v. 5, n. 8, p. 90-105, 1997.

MORACO, A. S. C. T. Um estudo sobre os conhecimentos geométricos adquiridos por alunos do Ensino Médio. 2006. 106f. Dissertação (Mestrado em Ensino de Ciências e Matemática) - Faculdade de Ciências, Universidade Estadual Paulista, Bauru, 2006.

NACARATO, A. M. Eu trabalho primeiro no concreto. Revista de Educação Matemática, São Paulo, v. 9, n. 9-10, p. 1-6, 2005.

PAIS, L. C. Didática da matemática: uma análise da influência francesa. 2. ed. Belo Horizonte: Autêntica, 2002.

PASSOS, C. L. B. Representações, interpretações e prática pedagógica: a geometria na sala de aula. 2000. 348f. Tese (Doutorado em Educação Matemática) - Faculdade de Educação, Universidade Estadual de Campinas, Campinas, 2000.

PAVANELLO, M. R.; ANDRADE, R. N. G. Formar professores para ensinar geometria: um desafio para as licenciaturas em matemática. Educação Matemática em Revista, São Paulo, v. 9, n. 11, p. 78-87, 2002.

PIROLA, N. A. Um estudo sobre a formação dos conceitos de triângulo e paralelogramo em alunos de primeiro grau. 1995. 108f. Dissertação (Mestrado em Psicologia Educacional) - Faculdade de Educação, Universidade Estadual de Campinas, Campinas, 1995. 
Proença, M. C.; Pirola, N. A.

PIROLA, N. A.; BRITO, M. R. F. A formação dos conceitos de triângulo e de paralelogramo em alunos da escola elementar. In: BRITO, M. R. F. (Org.). Psicologia da Educação Matemática: teoria e pesquisa. Florianópolis: Insular, 2001. p. 85-106.

PROENÇA, M. C. Um estudo exploratório sobre a formação conceitual em geometria de alunos do Ensino Médio. 2008. 200f. Dissertação (Mestrado em Ensino de Ciências e Matemática) - Faculdade de Ciências, Universidade Estadual Paulista, Bauru, 2008.

PROENÇA, M. C.; PIROLA, N. A. A formação conceitual em geometria: uma análise sobre polígonos e poliedros. In: SEMINÁRIO INTERNACIONAL DE PESQUISA EM EDUCAÇÃO MATEMÁTICA, 3., 2006, Águas de Lindóia. Anais... Águas de Lindóia: SBEM, 2006. 1 cd-rom.

. Um estudo sobre a formação de conceitos de polígonos e poliedros apresentada por alunos do $2^{\circ}$ ciclo do Ensino Fundamental e Ensino Médio. Revista de Educação Matemática, São Paulo, v. 10, n. 12, p. 51-58, 2007.

PROENÇA, M. C.; PIROLA, N. A.; QUINTILIANO, L. C. Um estudo sobre o desempenho de alunos do ensino médio em tarefas envolvendo o conceito de polígono e poliedro. In: SEMINÁRIO INTERNACIONAL DE PESQUISA EM EDUCAÇÃO MATEMÁTICA, 2., 2003, Santos. Anais... Santos: SBEM, 2003. 1 cd-rom.

QUINTILIANO, L. C. Conhecimento declarativo e de procedimento na solução de problemas algébricos. 2005. 159f. Dissertação (Mestrado em Educação Matemática) Faculdade de Educação, Universidade Estadual de Campinas, Campinas, 2005.

REZI, V. Um estudo exploratório sobre os componentes das habilidades matemáticas presentes no pensamento em geometria. 2001. 174f. Dissertação (Mestrado em Educação Matemática) - Faculdade de Educação, Universidade Estadual de Campinas, Campinas, 2001.

RIGODANZO, M.; ANGELO, C. L. Uma experiência de transposição didática com o cabrigéomètre II. Educação Matemática em Revista, São Paulo, v. 11, n. 16, p. 16-24, 2004.

SÃO PAULO (Estado). Proposta curricular de matemática. São Paulo: Secretaria de Estado de Educação, 2008.

Proposta curricular para o ensino de matemática: $2^{\circ}$ grau. São Paulo: Secretaria da Educação, CENP, 1992.

. Proposta curricular para o ensino de matemática: $1^{\circ}$ grau. São Paulo: Secretaria da Educação, CENP, 1997.

Sistema de avaliação do rendimento escolar do estado de São Paulo SARESP. São Paulo: Secretaria de Estado da Educação, 2007. Disponível em: <http:// saresp.fde.sp.gov.br/2007>. Acesso em: 15 maio 2008.

SCHEIDE, T. J. F. O papel da história da matemática no processo de ensino e aprendizagem. In: SEMINÁRIO INTERNACIONAL DE PESQUISA EM EDUCAÇÃO MATEMÁTICA, 3., 2006, Águas de Lindóia. Anais... Águas de Lindóia: SBEM, 2006. 1 cdrom. 
O conhecimento de polígonos e poliedros: ...

SILVA, C. M. Uso do logo em sala de aula: desempenho em geometria e atitudes em relação à matemática. 2003. 259f. Dissertação (Mestrado em Educação Matemática) Faculdade de Educação, Universidade Estadual de Campinas, Campinas, 2003.

SILVEIRA, M. R. A. O conceito em matemática e seus contextos. Educação Matemática em Revista, São Paulo, v. 13, n. 20/21, p. 47-58, 2006.

SORMANI JUNIOR, C. Um estudo exploratório sobre o uso da informática na resolução de problemas trigonométricos. 2006. 226f. Dissertação (Mestrado em Ensino de Ciências e Matemática) - Faculdade de Ciências, Universidade Estadual Paulista, Bauru, 2006.

ZABALA, A. A prática educativa: como ensinar. Porto Alegre: ArtMed, 1998.

ZUIN, E. S. L. Régua e compasso nos livros didáticos de matemática: o início de uma nova história na prática escolar. In: SEMINÁRIO INTERNACIONAL DE PESQUISA EM EDUCAÇÃO MATEMÁTICA, 2., 2003, Santos. Anais... Santos: SBEM, 2003. 1 cd-rom.

Artigo recebido em novembro de 2010 e aceito em fevereiro de 2011. 